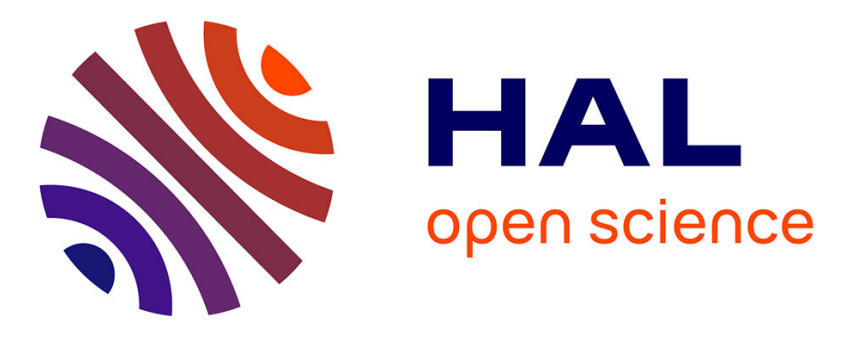

\title{
Mahaut d'Artois, une femme de pouvoir (1302-1329)
}

Christelle Balouzat-Loubet

\section{To cite this version:}

Christelle Balouzat-Loubet. Mahaut d'Artois, une femme de pouvoir (1302-1329). Antoine Destemberg; Yann Potin; Emilie Rosenblieh. Faire jeunesses, rendre justice, 3, Publications de la Sorbonne, pp.173-183, 2015, Série du Lamop, 978-2-85944-911-7. 10.4000/books.psorbonne.26310 . halshs01175899

\section{HAL Id: halshs-01175899 \\ https://shs.hal.science/halshs-01175899}

Submitted on 13 Jul 2015

HAL is a multi-disciplinary open access archive for the deposit and dissemination of scientific research documents, whether they are published or not. The documents may come from teaching and research institutions in France or abroad, or from public or private research centers.
L'archive ouverte pluridisciplinaire HAL, est destinée au dépôt et à la diffusion de documents scientifiques de niveau recherche, publiés ou non, émanant des établissements d'enseignement et de recherche français ou étrangers, des laboratoires publics ou privés. 


\title{
Mahaut d'Artois, une femme de pouvoir (1302-1329)
}

\author{
Christelle Balouzat-Loubet
}

$\mathrm{L}$ a comtesse Mahaut, qui dirige l'Artois pendant près de trente ans, reste aujourd'hui un personnage méconnu, popularisé par les écrits de Maurice Druon et leurs adaptations télévisées successives, mais longtemps oublié des travaux scientifiques. Les sources, très abondantes, permettent pourtant de dresser le portrait d'une personnalité curieuse, appartenant au milieu cultivé de la cour et exerçant un mécénat particulièrement actif. Elles montrent aussi une femme obstinée, qui résiste durant quatre années aux nobles artésiens révoltés et sort blanchie d'une dangereuse accusation d'empoisonnement. Petite-nièce, marraine et belle-mère de roi, princesse apanagée, membre du Conseil royal, pair de France, la comtesse d'Artois est une figure féminine qui a marqué l'histoire des derniers Capétiens. Étudier Mahaut d'Artois, c'est donc s'intéresser au parcours d'une femme en politique dans les premières années du XIV siècle. Certes, la comtesse a dû pour s'imposer à la tête de son apanage gouverner comme un homme, mais nous nous demanderons si cette grille de lecture est suffisante et si nous ne pouvons pas dégager de son règne des spécificités du pouvoir féminin.

Si Mahaut est un prince comme un autre, cela tient avant tout à sa formation et sa culture, que l'on pourrait qualifier de masculines.

C'est ce que suggère la composition de sa bibliothèque, dont le contenu peut être en partie reconstitué grâce à la comptabilité de l'Hôtel, les testaments de la comtesse et un acte dressant la liste des biens qui lui ont été volés lors de la révolte nobiliaire ${ }^{1}$. Ce dernier document mentionne ainsi la disparition de « 12 romans que granz que petiz, c'est assavoir : 3 rommans de Trytan de quoi l'en a recouvert l'un, un romans des faiz d'outremer, un romans des Enfances Ogier, un romans de l'ordenance mestre Tranque, un 1530, Paris, Promodis/Éditions du Cercle de la Librairie, 1989. 
romant de Renart, un romant des coustumes de Normandie, un romant de la Vyoleite petit, la Bible en rommans, la quele Madame a recouvree, un rommant des vies des sains, et un rommant du grant Kan tout ou pris, sanz les recouvrez, de $200 \AA^{2} »$. Les sources comptables mentionnent par ailleurs une Histoire de Troyes, un Roman de Perceval le Gallois ${ }^{3}$, et les Vœux du Paon ${ }^{4}$. La composition de cette bibliothèque reflète à la fois les préférences personnelles de la comtesse et les goûts collectifs de l'époque. Plusieurs titres témoignent d'un intérêt pour la littérature courtoise : la comtesse d'Artois ne possède rien moins que trois exemplaires de la Légende de Tristan, l'un des plus grands succès littéraires de l'époque médiévale. Sa bibliothèque abrite un exemplaire des Enfances Ogier, œuvre d'Adenet le Roi qui, à la fin du XIII ${ }^{\mathrm{e}}$ siècle, a également composé pour Marie de Brabant son Cleomadès. Mahaut possède aussi une copie du Roman de la Violette ou de Gérard de Nevers, rédigé entre 1227 et 1229 par Gerbert de Montreuil et dédié à Marie, comtesse de Ponthieu. Le Roman de Renart complète cet ensemble d'ouvrages divertissants. Mahaut, cédant comme la cour de France à la mode de la littérature orientale, s'offre aussi les écrits de Marco Polo, le Roman du Grand Khan, et un romans des faiz d'outremer 5 . Il est également notable que la comtesse soit l'une des rares à possèder dès 1305 un exemplaire des Chroniques des Rois de France, qu'elle fait enluminer à cette date en même temps que la version abrégée de l'ouvrage ${ }^{6}$. Elle montre ainsi l'intérêt qu'elle porte à l'œuvre de compilation historiographique initiée

2. Antoine Le Roux de Lincy, «Inventaires des biens meubles et immeubles de la comtesse Mahaut d'Artois pillés par l'armée de son neveu en 1313 [1316] ", Bibliothèque de l'École des chartes, $3^{\text {e }}$ série, t. 3, 1852, p. 63. L'un des exemplaires de la Légende de Tristan a été acheté en 1310 pour sept livres parisis : Dainville, AD Pas-de-Calais, A 275 ; Chrétien Dehaisnes, Documents et extraits divers concernant l'histoire de l'art dans la Flandre, l'Artois et le Hainaut avant le XVe siècle, $1^{\mathrm{re}}$ partie : 627-1373, Lille, Impr. L. Danel, 1886, p. 191 ; Jules-Marie Richard, Une petite nièce de saint Louis : Mahaut, comtesse d'Artois et de Bourgogne (1302-1329). Étude sur la vie privée, les arts et l'industrie, en Artois et à Paris au commencement du XIVe siècle, Paris, Honoré Champion, 1887, p. 101. Le Roman du Grand Khan est mentionné dans les comptes de l'Hôtel de la Toussaint 1312 : Dainville, AD Pasde-Calais, A 298; Richard, op. cit.

3. 30 novembre 1308, Dainville, AD Pas-de-Calais, A 244 ou A $244^{41}$; cf. aussi Richard, op. cit., n. 4 p. 100 et Chrétien Dehaisnes, op. cit., p. 183.

4. Dainville, AD Pas-de-Calais, A 312 ; Richard, op. cit., n. 3 p. 102.

5. Il s'agit d'une œuvre consacrée aux croisades, que le titre ne permet pas d'identifier précisément. Tout au plus peut-on supposer qu'il s'agit d'une version de l'ouvrage de Guillaume de Tyr, l'Historia rerum in partibus transmarinis gestarum.

6. 1305, Dainville, AD Pas-de-Calais, A 399. 
par Louis IX et poursuivie par Philippe le Bel, tout en reprenant à son compte le mythe des origines troyennes de la dynastie ${ }^{7}$.

Éduquée comme un garçon, adepte des mêmes lectures, Mahaut a une pratique du gouvernement comparable à celle des princes contemporains, avec lesquels elle partage ses équipes de pouvoir : elle confie ses intérêts à des hommes renommés qui ne sont pas à son service exclusif mais mettent leur talent au service de différents maîtres, dont le roi.

Pierre de Maucreux, avocat pensionnaire de la comtesse au Parlement de Paris, auteur, avec son frère Guillaume de Maucreux, d'un traité de droit intitulé Les ordonnances de plaidoier de bouche et par escript abbregiés par Pierre et Guillaume Maucrueuls de Montagu, selon droit, us et coustume, le ressort est en Parlement cujus est questio ${ }^{8}$, est aussi avocat du roi au Parlement depuis le 21 mars 1324. En 1327, il fait office d'arbitre au Parlement avec Guillaume du Breuil ${ }^{9}$. En 1328, il devient avocat au Parlement pour les échevins de Reims et, après le décès de Mahaut, plaide pour Robert d'Artois aux côtés de Guillaume du Breuil. Il est encore en activité en mars 1332, le connétable Raoul de Brienne le charge alors avec Jean l'Orfèvre de négocier un accord avec les représentants du comte d'Auxerre, à savoir Eudes de Sens et Hugues de Crusy. Peu après, il est désigné par les capitouls de Toulouse pour être leur avocat. Le 20 mai 1335, il est fait état de son décès ${ }^{10}$. Jean Hanière, qui doit une bonne part de sa célébrité à son statut d'accusateur dans le procès d'Enguerrand de Marigny, est cité comme avocat de Mahaut au Parlement de Paris en 1317 et 1322. Il devient conseiller du roi à partir de $1335^{11}$.

Mahaut emploie ainsi quelques-uns des meilleurs juristes et hommes de la pratique de l'époque. Elle peut aussi compter sur Thierry de Hérisson, son principal conseiller et garde des Sceaux.

7. Le Roman des rois, composé en français à la demande de Louis IX, est offert par Primat à Philippe III en 1274. Il relate l'histoire des Francs et de leur roi depuis les origines troyennes jusqu'à la mort de Philippe Auguste en 1223. On ne conserve qu'une vingtaine de manuscrits des Grandes chroniques de France dans leurs différentes versions antérieures à 1375 : Isabelle Guyot-Bachy et Jean-Marie Moeglin, "Comment ont été continuées les Grandes Chroniques de France dans la première moitié du XIV siècle ", Bibliothèque de l'École des chartes, 163-2, 2005, p. 385-433.

8. Paris, BnF, fr. 19832 : Félix Aubert, "Les sources de la procédure au Parlement au XIVe siècle ", Bibliothèque de l'École des chartes, 76-1, 1915, p. 521-525.

9. Actes du Parlement de Paris, première série (1254-1328), éd. par E. Boutaric, t. 2 (1299-1328), Paris, H. Plon, $1867, \mathrm{n}^{\circ} 7988$.

10. Aubert, op. cit., p. 517-520.

11. Roland Delachanal, Histoire des avocats au Parlement de Paris (1300-1600), Paris, Plon, 1885, p. 356-357. 
Titulaire d'une prébende canoniale dans sa ville d'origine, il est signalé comme clerc du comte d'Artois dès 1293 . Resté au service de Mahaut, il en devient rapidement le représentant officiel : en 1315, il porte le titre de lieutenant de la comtesse $\mathrm{e}^{12}$. C'est sans doute à ce titre que le receveur d'Artois lui verse deux cents livres de gages à chaque terme ${ }^{13}$, des indemnités très élevées par rapport à celles des autres officiers comtaux ${ }^{14}$. Il joue un rôle essentiel dans le contrôle des comptes de la comtesse, qui l'autorise même à ordonner des paiements en son nom. Même s'il n'en porte jamais le titre, il est le chancelier de Mahaut, comme le montre un acte de février 1303 dans lequel la comtesse précise que «li dis maistres Thierrys ou tans que ces lettres furent faites portoit nos grans seaus ${ }^{15}$ ». En février 1308 , c'est encore à Thierry qu'il revient d'apposer le sceau comtal en bas des documents officiels ${ }^{16}$. D'autres indices témoignent de son importance dans le gouvernement du comté : destinataire de $24 \%$ des messages envoyés par Mahaut entre 1314 et 1322, il utilise le personnel du bailliage d'Arras pour correspondre avec la fille de Mahaut, les membres du Conseil comtal ou encore les agents royaux ${ }^{17}$. Tout en servant la dynastie comtale, il poursuit une carrière au service de la monarchie : régulièrement qualifié de «clerc du roi » à partir de $1300^{18}$, il participe au Parlement en 1319 et porte le titre de " conseiller du roi " dans un acte du 9 mai $1321^{19}$. Homme d'Église, il accumule de nombreuses prébendes canoniales. Sans doute favorisée par le soutien, voire les interventions de la comtesse d'Artois, cette carrière ecclésiastique est couronnée par son accession à l'épiscopat d'Arras en 1327.

Comme les rois, la comtesse se choisit donc un favori, qu'elle comble de cadeaux et dont elle assure l'ascension sociale. Comme eux, elle cherche aussi à manifester son pouvoir et sa puissance aux yeux du plus grand nombre.

12. S.d. [28 octobre-15 novembre 1315], Dainville, AD Pas-de-Calais, A $61^{23}$.

13. Saint-Omer, BM, ms 871, fol. 9 (Chandeleur 1312), fol. 23 (Toussaint 1321), fol. 43 (Ascension 1322), fol. 59 (Chandeleur 1328).

14. Selon le compte de l'Ascension 1322, les procureurs et avocats comtaux au Parlement de Paris touchent entre vingt et quarante livres tournois de pension pour une année (ibid., fol. 43). 15. Paris, BnF, nouv. acq. fr. 21199, fol. $7 \mathrm{n}^{\circ} 151$.

16. 19 février 1308, Paris, BnF, nouv. acq. fr. 21199, fol. 2, nº 68.

17. Comptes du bailliage d'Arras (CbA), Toussaint 1304, Dainville, AD Pas-de-Calais, A 200² ; CbA, Toussaint 1312, ibid. A $294^{2}$.

18. La première mention dans les sources artésiennes date du 5 novembre 1300 (Dainville, AD Pas-de-Calais, A 161).

19. Olivier Canteaut, Gouvernement et hommes de gouvernement sous les derniers Capétiens (1313-1328), thèse de doctorat dactylographiée, sous la direction de Claude Gauvard, université Paris 1 Panthéon-Sorbonne, 2005 , 3 vol., n. 37 p. 10 et p. 37. 
Cela passe par exemple par la possession d'une ménagerie, caractéristique des grandes résidences princières. Ainsi, au XII ${ }^{\mathrm{e}}$ siècle, de nombreuses cours européennes en entretiennent une : on trouve ainsi des lions, des léopards, des lynx, des chameaux et un porc-épic dans le parc royal de Woodstock ${ }^{20}$; Louis IX a des lions, un porc-épic et un éléphant; Philippe le Bel des lions, des lionnes, des léopards et un ours blanc; les rois de Navarre un lion, une lionne, une autruche et un chameau; René d'Anjou des chameaux, un éléphant, des lions, des tigres et des ours ${ }^{21}$. Au début du XIV ${ }^{e}$ siècle, Mahaut possède un castor, un sanglier, un ours, des chats sauvages, des loups et des oiseaux de proie nordiques ou méditerranéens ${ }^{22}$. Bien que moins prestigieuse que celle des rois de France ou d'Angleterre, la ménagerie comtale reste une autre façon de manifester le pouvoir de la comtesse sur les animaux, dont la cohabitation est une image du Paradis dans lequel toutes les espèces animales vivaient en harmonie sous la direction de Dieu ${ }^{23}$. L'inscription dans l'espace du pouvoir comtal passe aussi par l'expression de la piété comtale, qui obéit aux normes des milieux princiers contemporains : durant son règne, Mahaut restaure, construit ou s'associe à la fondation de plusieurs abbayes et hôpitaux, à Hesdin, Arras, Saint-Omer ou Gosnay; elle organise par ailleurs un véritable culte des morts, qui se manifeste par la fondation d'obits, mais aussi par l'édification de riches tombeaux, qui célèbrent la dynastie et en soulignent le prestige. Son père, Robert II, est inhumé à Maubuisson ; son époux, Othon IV, repose à l'abbaye de Cherlieu, en Bourgogne; les gisants de ses fils Jean et Robert se trouvent respectivement à l'abbaye bourguignonne de Poligny et aux Cordeliers, à Paris.

Rien ne semble donc distinguer la comtesse d'Artois des autres princes contemporains, dont elle partage les références culturelles et les méthodes de gouvernement. Plusieurs points incitent néanmoins à nuancer cette première impression.

Tout d'abord, bien qu'elle évolue dans un milieu essentiellement masculin, Mahaut entretient des relations suivies avec d'autres femmes de pouvoir, telles Marie de Brabant ou Jeanne de Bourgogne, qui influencent sans doute son gouvernement.

En grande partie élevée à la cour de France, Mahaut semble avoir tissé avec la veuve du roi Philippe III des liens particulièrement forts. Les comptes de

20. François Duceppe-Lamarre, "Une réserve spectaculaire, les parcs à gibier ", dans "Forêts et réserves ", Cahier d'études Environnement, forêt et société, XVI'-XXe siècle, 13, 2003, p. 13.

21. Lexikon des Mittelalter, Verlag J. B. Metzler, vol. 9, col. 117-119.

22. Duceppe-Lamarre, op cit.

23. Lexikon des Mittelalter, ibid. 
l'Hôtel témoignent des relations suivies qu'entretiennent les deux femmes : entre la Chandeleur 1314 et l'Ascension 1322, $6 \%$ des messages envoyés par Mahaut lui sont destinés, c'est-à-dire autant qu'au roi Philippe V (7\%) et plus qu'à certains membres de sa famille, comme Hugues de Bourgogne ( $4 \%$ ). La comtesse d'Artois rencontre aussi la reine lors de ses séjours parisiens, en particulier à l'abbaye royale de Poissy, où cette dernière s'est retirée ${ }^{24}$. Quant à sa fille aînée, Jeanne, elle est la destinataire de $27 \%$ des messages envoyés entre 1314 et 1322 . Il s'agit dans un premier temps d'une correspondance exclusivement personnelle. Puis, de 1316 à 1322, Jeanne est reine de France. Il est donc probable qu'à cette période les missives échangées entre les deux femmes traitent aussi bien d'affaires privées que de politique.

Mahaut d'Artois semble d'ailleurs particulièrement soucieuse de marquer son attachement au rôle politique des femmes.

C'est ce que montre le décor de sa chambre du château d'Hesdin. La pièce est ornée d'une rangée de têtes de rois moulées en plâtre à l'image des souverains français, et de fleurs de lys d'or en plomb ou étain verni fixées sur des murs peints d'azur. À compter de 1307, les reines figurent en bonne place auprès de leurs époux : sans doute Mahaut tient-elle ainsi à affirmer le rôle essentiel des femmes dans l'histoire dynastique, alors même que les problèmes de succession par les femmes sont au cœur de la vie politique. Effectivement, la comtesse d'Artois doit s'imposer dans un contexte particulièrement difficile pour les femmes : dès 1314 , la règle de masculinité est introduite dans la succession de l'apanage de Poitiers ${ }^{25}$, et, en 1316 , la fille de Louis X, Jeanne de France, est écartée du trône de France au profit de Philippe V. Cela encourage sans doute son neveu Robert à réclamer l'héritage dont il s'estime spolié. C'est en 1307, alors qu'il vient d'atteindre sa majorité, qu'il attaque pour la première fois Mahaut, avec l'appui de sa mère Blanche de Bretagne. Ce n'est que le premier de trois procès, les deux autres débutant en 1316 et 1329 . Le règlement du conflit en faveur de Mahaut montre que la transmission des apanages bénéficie encore d'un flou juridique qui permet à chaque roi d'agir au cas par cas, selon ses intérêts et témoigne de la puissance de la comtesse qui parvient à s'imposer dans ce contexte défavorable.

24. Mahaut séjourne dans le prieuré de Dominicaines les 12 et 13 janvier 1314 (Comptes de l'Hôtel [CH], Chandeleur 1314, Dainville, AD Pas-de-Calais, A 316 fol. 10), du 24 au 29 mai 1319 (CH, Toussaint 1319, Dainville, AD Pas-de-Calais, A 374 fol. $3 \mathrm{v}^{\circ}$ ), les 14 et 15 décembre 1319 (CH, Chandeleur 1320, Dainville, AD Pas-de-Calais, A 378 fol. $5 \mathrm{v}^{\circ}$ ), les 4 et 5 juillet 1321 (CH, Toussaint 1321, Dainville, AD Pas-de-Calais, A 396 fol. $3 \mathrm{v}^{\circ}$ ).

25. 29 novembre 1314, Paris, AN, J $390 \mathrm{n}^{\circ} 10$; Jean Favier, Un conseiller de Philippe le Bel : Enguerrand de Marigny, Paris, -, 1963, p. 232. 
Si ces procès successifs constituent la menace la plus grave qui pèse sur l'héritage de Mahaut, ce n'est cependant pas la seule. Entre 1314 et 1319, cette dernière doit faire face à une révolte particulièrement virulente de la noblesse artésienne.

Celle-ci réclame le respect des coutumes artésiennes, l'annulation des nouvelletés et l'ouverture de deux enquêtes, l'une sur les agissements de ses officiers, l'autre sur les nouvelles garennes ${ }^{26}$. L'obstination de la comtesse, qui refuse de plier face aux exigences des révoltés et tarde à leur donner satisfaction, envenime rapidement la situation. Ces derniers oublient quelque peu leurs premières revendications pour s'en prendre plus directement à la comtesse et son gouvernement. Les violences se multiplient, à la fois contre les biens et les personnes. Mahaut quitte alors son domaine qu'elle confie à son fils Robert et en appelle au roi, qui fait passer le comté sous sa main. En 1317, la comtesse est soupçonnée d'avoir empoisonné le roi Louis $\mathrm{X}$ : le fait que la comtesse soit une femme sert sans doute les révoltés qui font appel dans l'imaginaire collectif à l'image de la sorcière et de l'empoisonneuse ${ }^{27}$. Est-ce à dire que la révolte se nourrit du fait que l'autorité soit exercée par une femme? De fait, un pouvoir féminin pourrait être plus difficile à supporter pour la noblesse artésienne parce que Mahaut est bien plus présente que ses ancêtres : son père Robert II était un comte absent, régulièrement appelé par l'ost royal et qui a séjourné plusieurs années en Italie. Mais ces multiples attaques peuvent aussi être symptomatiques d'une fragilité relative de Mahaut, imputable à son statut de femme.

Cette dernière hypothèse se justifie pleinement lorsque l'on considère les efforts fournis par Mahaut, tout au long de son règne, pour assoir sa légitimité.

Elle ne cesse ainsi de rappeler ses liens avec la dynastie capétienne, dont elle fait apposer les armes sur sa vaisselle ou sur les murs de ses résidences. Si Mahaut revendique cette filiation, c'est qu'elle peut en tirer un immense prestige, prestige dû au titre royal de Louis IX mais plus encore à sa canonisation en 1297. En 1329, elle acquiert ainsi auprès d'Étienne de Salins, pour sa chapelle, une image de saint Louis avec deux anges, faite d'argent, d'or,

26.16 octobre 1315, Dainville, AD Pas-de-Calais, A 6033 et A 60 ${ }^{34}$; André Artonne, Le mouvement de 1314 et les chartes provinciales de 1315, Paris, F. Alcan, 1912, P. J. n 16, p. 179-181.

27. Les accusations portées par Isabelle de Feriennes entraînent l'ouverture d'un procès qui ne s'achève que le 9 octobre 1317, avec un arrêt disculpant Mahaut (9 octobre 1317, Paris, BnF, nouv. acq. fr. 20025, pièce 76 ; Denis-Charles de Godefroy de Ménilglaise, " Mahaud comtesse d'Artois-Accusation de sortilège et d'empoisonnement-Arrêt d'absolution-Confédération des nobles du Nord de la France ", Mémoires de la société Impériale des Antiquaires de France, 8, 1865, p. 195-218). 
d'émail, de perles et de pierres. En 1310, elle offre à Notre-Dame de Paris un vaisseau d'argent à l'occasion de la saint Louis et, en 1324, elle fait don de deux statuettes du saint à l'abbaye de la Thieulloye ${ }^{28}$. Elle rend également hommage à son illustre ancêtre en donnant son nom à la chapelle du château d'Hesdin ${ }^{29}$. Elle est l'une des premières à posséder des Heures de saint Louis, commandées en 1327 à un libraire parisien ${ }^{30}$. Elle détient aussi des reliques du saint, dérobées lors du sac du château d'Hesdin en 1316 : l'inventaire des biens pillés, établi en 1317, mentionne la perte d'une "image de St Loys a tout 2 angeleiz [...] touz seaus seur un pié et tout d'argent doré, ou pris de $300 £$ sanz le saintuaire, que en ne peut estimer ${ }^{31}$ ". Comme la plupart des princesses de sang royal, Mahaut conserve également des fragments des reliques de la Passion, mais elle est une des rares, avec Jeanne d'Évreux, à posséder ces fragments de reliques du roi saint ${ }^{32}$. La fidélité de Mahaut à la mémoire de saint Louis influence également ses pratiques religieuses et plus particulièrement sa manière d'exercer la charité. Comme son illustre ancêtre, la comtesse d'Artois manifeste un grand intérêt pour les ordres mendiants, qu'elle comble de nombreux dons. Ce choix apparaît très clairement dans son troisième et dernier testament : elle lègue alors vingt livres aux Franciscains et Dominicains de Paris; cent sous chacun aux Ermites de saint-Augustin, aux Carmes, aux Croisiers et aux Guillemins de Paris; soixante sous aux Trinitaires ; dix livres aux clarisses de Longchamp ; cent sous au couvent de Saint-Marcel près de Paris. Elle fonde son obit au couvent des Franciscains et au couvent des Prêcheurs, à Paris. Elle leur lègue respectivement vingt et dix livres pour pitance ce jour-là. Elle prévoit aussi des dons destinés aux premiers chapitres généraux suivant sa mort pour les ordres des Dominicains, des Franciscains, des ermites de saint Augustin et des Carmes. Elle demande que chaque couvent célèbre pour elle une messe solennelle des défunts précédée des vigiles à neuf leçons, que chaque frère prêtre célèbre une messe pour elle et que les autres frères disent les sept psaumes de la pénitence avec la litanie. Enfin, elle lègue au couvent franciscain de Saint-Omer deux croix

28. Dainville, AD Pas-de-Calais, A 270; Richard, op. cit., n. 2 p. 240 ; 13 décembre 1324, Dehaisnes, op. cit., p. 259.

29. Christian de Mérindol, «Le décor peint et armorié en France à l'époque médiévale : les châteaux et résidences des comtes d'Artois. Bilan et perspectives ", dans Liber amicorum Raphaël de Smedt, vol. 2 (Miscellanea neerlandica, XXV), Louvain-Paris, Peeters, 2001, p. 7.

30. Allirot, op. cit., p. 191.

31. Le Roux de Lincy, op. cit., p. 60.

32. Mahaut conserve une épine de la sainte Couronne dans un vaisseau d'argent portant une couronne, orné d'un angelot, de pierreries et d'or. L'ouvrage est l'œuvre d'un orfèvre arrageois, Jacques de Douai : Allirot, op. cit., p. 223 et 226. 
en or et aux clarisses de Saint-Omer une autre d'argent doré. Il faut rappeler également que le monastère qu'elle fonde à la Thieulloye en 1323 et qu'elle ne manque pas de citer dans son testament est un couvent de dominicaines. Elle entreprend aussi, en 1322, la reconstruction du couvent des clarisses de SaintOmer, fondé par son père et pourvoit à l'avenir des religieuses. Son confesseur lui-même, Thomas de Besançon, est un dominicain. Deux autres frères Prêcheurs sont avec lui les exécuteurs testamentaires de Mahaut ${ }^{33}$. Outre cette sensibilité au message des mendiants, la comtesse d'Artois partage avec saint Louis la pratique du mandé, ce rituel du lavement des pieds que le roi a généralisé et institutionnalisé. Les comptes de l'Hôtel suggèrent qu'elle le pratique au moins une fois par an tout au long de son règne ${ }^{34}$.

Cet attachement à la mémoire de saint Louis et, plus largement, à la dynastie capétienne, transparaît également dans le choix des lieux de sépulture.

Lorsqu'elle dicte son troisième testament, en 1329, la comtesse d'Artois a obtenu du pape l'autorisation de diviser sa sépulture. Selon ses dernières volontés, son corps doit être inhumé à Maubuisson, auprès de son père, et son cœur aux Cordeliers, auprès de son fils Robert ${ }^{35}$. L'abbaye de Maubuisson, fondée par la mère de saint Louis, Blanche de Castille, est au XIV siècle la nécropole des femmes de sang royal et de quelques princes capétiens dont les sépultures ne peuvent reposer à Saint-Denis. Les Cordeliers de Paris abritent également les tombeaux de plusieurs reines et filles de France, comme Blanche, fille de saint Louis, ou Marie de France, fille de Philippe VI ${ }^{36}$. Les gisants sont une dernière occasion de graver dans la pierre l'écu d'Artois et les armes de France.

La proximité avec la dynastie capétienne est donc culturelle, spirituelle, mais aussi géographique : Mahaut d'Artois est une princesse plus parisienne qu'artésienne, qui réside souvent près du roi.

De son père, elle hérite en Île-de-France de deux résidences, à savoir l'hôtel d'Artois situé rue Mauconseil, à Paris, et le manoir de Conflans, tout proche de la résidence royale de Vincennes. La localisation de ces demeures comtales est manifestement guidée par la proximité du souverain : l'hôtel d'Artois est

33. 24 mars 1329, Bernard Delmaire, "La comtesse Mahaut d'Artois et ses trois testaments $(1307,1318,1329) "$ " Histoire et archéologie du Pas-de-Calais : bulletin de la commission départementale d'histoire et d'archéologie du Pas-de-Calais, 23, 2005, p. 36 [8] et [11], p. 37 [15], [16] et [17], p. 38 [20] et [22].

34. Priscille Aladjidi, Rex pater pauperum. Théorie et pratique de la charité royale en France (XIII ${ }^{e-}$ $\mathrm{XV}^{e}$ siècles), thèse de doctorat dactylographiée sous la direction de Colette Beaune, université Paris X Nanterre, 2006, 2 t., p. 607.

35. 24 mars 1329, Delmaire, art. cité, p. 35 [2].

36. Allirot, op. cit., n. 36 p. 239. 
à environ un kilomètre du Palais de la Cité, et le manoir de Conflans se trouve à une distance comparable du château du roi à Vincennes. Les itinéraires comparés de Mahaut et de Philippe IV le Bel montrent que celle-ci s'arrange pour séjourner au plus près du souverain. En janvier 1310, avertie par quelque messager de l'arrivée imminente du roi à Paris, elle arrive dans la capitale un jour seulement après lui. En octobre 1310, elle se trouve avec lui à l'abbaye Saint-Denis : Philippe y est du 6 au 9, Mahaut du 5 au 9. Elle choisit ensuite de s'installer à Conflans, à côté de Charenton-le-Pont, où se trouve la cour royale à partir du $12^{37}$. Ce voisinage lui permet de multiplier les audiences avec le souverain, généralement pour traiter d'affaires importantes, qu'elle gère personnellement. L'accord scellant la scession par Mahaut à Philippe de tout ce qu'elle possède dans le comté de Bourgogne est ainsi signé les 4 et 5 novembre 1309 , alors que la comtesse se trouve avec la cour royale à Pierrefonds ${ }^{38}$. En octobre 1310, la chancellerie royale émet, au terme de la rencontre entre les deux cousins, des actes concernant d'une part l'assiette qu'elle a faite à Robert d'Artois, d'autre part les conventions conclues avec le roi au sujet des terres concédées à son deuxième fils dans le comté de Bourgogne ${ }^{39}$. En janvier 1310, Mahaut profite d'une entrevue avec le roi pour lui demander confirmation de ses lettres notifiant le contrat de mariage de Louis de Marigny avec Roberte de Beaumetz ${ }^{40}$. Lors de ses séjours franciliens, la comtesse peut aussi participer au Conseil du roi et, ainsi, prendre part à la vie politique du royaume. Elle en profite également pour afficher ses bonnes relations avec la famille royale. Entre 1314 et 1322, elle reçoit le roi et la reine, à Paris les 7 et 8 décembre 1316, à Conflans les 26 février et 18 mai 1318, le 22 janvier 1319, les 21, 25 et 26 juin 1319 et le 29 juin $1321^{41}$. Elle profite à son tour de l'hospitalité royale à Charenton, entre le 23 et le 30 avril $1322^{42}$. Le soutien de la famille royale est donc l'un des fondements de la légitimé comtale.

37. Élisabeth Lalou, Itinéraire de Philippe IV le Bel (1285-1314), vol. 2 « Routes et itinéraires ", Paris, Diffusion de Boccard (Mémoires de l'Académie des Inscriptions et Belles-Lettres, 37), 2007, p. 346 et $357-358$.

38. Dijon, AD Côte-d'Or, B 304 ; Lalou, op. cit., p. 341 et p. 357-358.

39. Paris, AN, JJ $45 \mathrm{n}^{\circ} 203$ et 204 ; Lalou, op. cit., p. 358.

40. Paris, AN, JJ $42^{\mathrm{B}} \mathrm{n}^{\circ} 189$; Lalou, ibid., p. 346 . Une nouvelle rencontre a peut-être lieu le 2 février suivant: Mahaut obtient ce jour une quittance per vos pour six mille livres petits tournois équivalant au premier terme des vingt-quatre mille livres qu'elle doit à son neveu Robert : Dainville, AD Pas-de-Calais, A 564; Lalou, op. cit., n. 1 p. 346.

41. CH, Chandeleur 1317, Dainville, AD Pas-de-Calais, A 351 fol. 4; CH, Ascension 1318, ibid. A 361 fol. $3 \mathrm{v}^{\circ}$ et fol. $9 \mathrm{v}^{\circ}$; CH, Chandeleur 1319, ibid. A 368 fol. 7 ; $\mathrm{CH}$, Toussaint 1319, ibid. A 374 fol. $4 V^{\circ}$ et fol. 5 ; CH, Toussaint 1321, ibid. A 396 fol. 3 .

42. $\mathrm{CH}$, Ascension 1322, ibid. A 403 fol. 13-14. 
Pour s'imposer pendant près de trente ans à la tête de la principauté artésienne, Mahaut a dû faire preuve de qualités qui sont celles de tout prince. Entourée d'équipes de pouvoir fidèles et compétentes, soucieuse de construire une mémoire de la dynastie comtale, elle gouverne comme un homme et résiste aux diverses attaques dont elle est la cible.

Son règne n'en reste pas moins marqué par son statut de femme : malgré une légitimité fragilisée en un temps où la tendance est à l'exclusion des femmes de la vie politique, la comtesse parvient à s'imposer à la tête de l'Artois tout en participant à l'affirmation du corps politique de la femme. C'est aussi en cela qu'elle est une personnalité d'exception. 


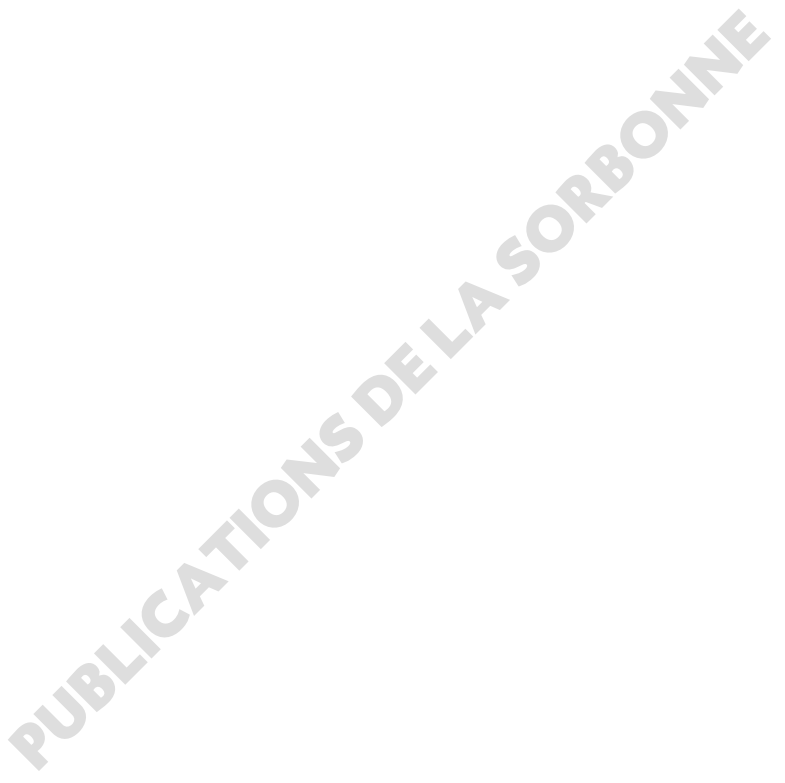

